

University of Central Florida

STARS

$1-1-2005$

\title{
Electron-beam-induced current and cathodoluminescence studies of thermally activated increase for carrier diffusion length and lifetime in n-type $\mathrm{ZnO}$
}

\author{
O. Lopatiuk \\ University of Central Florida \\ L. Chernyak \\ University of Central Florida
}
A. Osinsky
J. Q. Xie
P. P. Chow

Find similar works at: https://stars.library.ucf.edu/facultybib2000

University of Central Florida Libraries http://library.ucf.edu

This Article is brought to you for free and open access by the Faculty Bibliography at STARS. It has been accepted for inclusion in Faculty Bibliography 2000s by an authorized administrator of STARS. For more information, please contactSTARS@ucf.edu.

\section{Recommended Citation}

Lopatiuk, O.; Chernyak, L.; Osinsky, A.; Xie, J. Q.; and Chow, P. P., "Electron-beam-induced current and cathodoluminescence studies of thermally activated increase for carrier diffusion length and lifetime in $\mathrm{n}$ type ZnO" (2005). Faculty Bibliography 2000s. 5430.

https://stars.library.ucf.edu/facultybib2000/5430

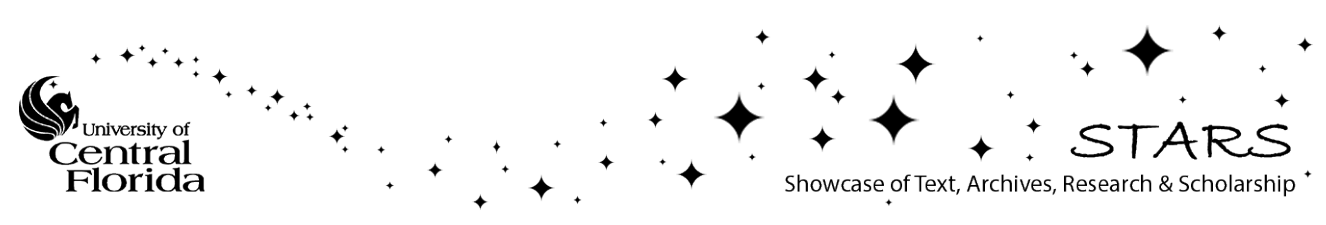




\title{
Electron-beam-induced current and cathodoluminescence studies of thermally activated increase for carrier diffusion length and lifetime in $n$-type ZnO
}

\author{
O. Lopatiuk and L. Chernyak ${ }^{\mathrm{a})}$ \\ Physics Department, University of Central Florida, Orlando, Florida 32816-2385
}

A. Osinsky, J. Q. Xie, and P. P. Chow

SVT Associates, Eden Prairie, Minnesota 55344

(Received 23 May 2005; accepted 25 August 2005; published online 11 October 2005)

\begin{abstract}
Temperature dependence of the minority carrier diffusion length and lifetime in bulk $n$-type $\mathrm{ZnO}$ was studied using electron-beam-induced current and cathodoluminescence techniques. The diffusion length was observed to increase exponentially over the temperature range from $25^{\circ} \mathrm{C}$ to $125^{\circ} \mathrm{C}$, yielding activation energy of $45 \pm 2 \mathrm{meV}$. Concomitant decrease of the cathodoluminescence intensity for the near-band-edge transition was also observed. The activation energy determined by optical measurements was $58 \pm 7 \mathrm{meV}$. The larger minority carrier diffusion length and smaller luminescence intensity are attributed to the increased lifetime of nonequilibrium holes in the valence band at elevated temperatures. (c) 2005 American Institute of Physics.
\end{abstract}

[DOI: $10.1063 / 1.2106001]$

While $\mathrm{ZnO}$ is suitable for many conventional wideband-gap semiconductor applications, its optical, electronic, and structural properties offer a significant advantage over other materials, such as $\mathrm{GaN}$ and $\mathrm{SiC}$. These characteristics include high exciton binding energy (60 meV, compared to $\sim 25 \mathrm{meV}$ in $\mathrm{GaN}$ ), transparency to visible radiation, availability of bulk single crystals for use as substrates in epitaxial growth, and possibility of wet chemical processing. ${ }^{1,2}$

Until recently, the applications potential of $\mathrm{ZnO}$ has been hindered by the lack of viable $p$-type material. While it is relatively easy to obtain $n-\mathrm{ZnO}$, introducing sufficiently shallow acceptor levels is notoriously problematic. Although several heterojunction light-emitting diodes with an $n-\mathrm{ZnO}$ active region have been demonstrated, ${ }^{3,4}$ the quality of such devices suffers due to inevitable crystallographic mismatch at the interface. This limitation is overcome by using all ZnO-based materials. Taking into account the new developments in growth techniques and progress in $p$-type doping of $\mathrm{ZnO}$, achieving quality, homojunction-based bipolar devices is likely to take place in the near future. In fact, fabrications of $\mathrm{Zn}_{0.9} \mathrm{Mg}_{0.1} \mathrm{O} / \mathrm{ZnO} p$ - $n$ junctions and $\mathrm{ZnO} p$ - $n$ homojunctions have already been reported. ${ }^{5}$

Since the functionality of bipolar devices is based on minority carrier transport, the investigation of minority carrier diffusion lengths and lifetimes in both $n$ - and $p$-type $\mathrm{ZnO}$, as well as in related materials, is especially timely. Moreover, considering possible applications in hightemperature electronics, the insight into the temperature dependence of $\mathrm{ZnO}$ minority carrier diffusion length and lifetime is of great value, especially given the fact that, to the best of our knowledge, the relevant publications on the subject are limited. Minority carrier diffusion length in $p$-type $\mathrm{ZnMgO}$ has been previously reported in Ref 6 . In this letter, we present variable-temperature electron-beam-induced current (EBIC) measurements for minority hole diffusion length in $n$-type $\mathrm{ZnO}$ and combine these measurements with

${ }^{a)}$ Electronic mail: chernyak@ physics.ucf.edu cathodoluminescence (CL) spectroscopy to show that temperature-induced changes in minority carrier transport are associated with increased carrier lifetime in the valence band.

The experiments were carried out on commercially available bulk $n$-type $\mathrm{ZnO}$ samples grown by hydrothermal technique. Room-temperature Hall measurements showed the samples to be weakly $n$ type with an electron concentration of $\sim 10^{14} \mathrm{~cm}^{-3}$ and a mobility of $\sim 150 \mathrm{~cm}^{2} / \mathrm{V} \mathrm{s}$. The samples under investigation were cleaved perpendicular to $c$ plane thus exposing a nonpolar $a$ plane of $\mathrm{ZnO}$. This was motivated by the observations that the latter crystallographic plane results in a better quality of Schottky contacts, as opposed to those deposited on the $c$ plane. Schottky barriers were, therefore, fabricated by electron-beam evaporation of a $100 \mathrm{~nm}$ thick Au layer on $\mathrm{ZnO} a$ plane and subsequent lift off. The quality of the Schottky contacts is crucial, as it is directly related to the magnitude of EBIC signal. The EBIC technique is the method of choice for determining the minority carrier diffusion length. It is based on measuring the current due to nonequilibrium carriers generated by the electron beam of the scanning electron microscope (SEM) and collected by the built-in field of the Schottky barrier. A detailed description of EBIC technique can be found elsewhere..$^{7-10}$

A series of variable-temperature EBIC experiments was performed in situ in a Phillips XL30 SEM fitted with a hot stage and an external temperature controller (Gatan). A pair of Au contacts with significantly different areas was used, with the larger area contact being pseudo-Ohmic. At each temperature, several measurements were taken by scanning the beam of the SEM along a line perpendicular to the edge of the Schottky contact, and recording the exponential decay of current. The EBIC signal was processed with a Stanford Research Systems SR570 preamplifier and a Keithley 2000 voltmeter; the data were recorded and fitted using specially designed software to extract the minority carrier diffusion length $(L)$. Upon performing a number of measurements, the mean value of $L$ and its standard deviation were determined 


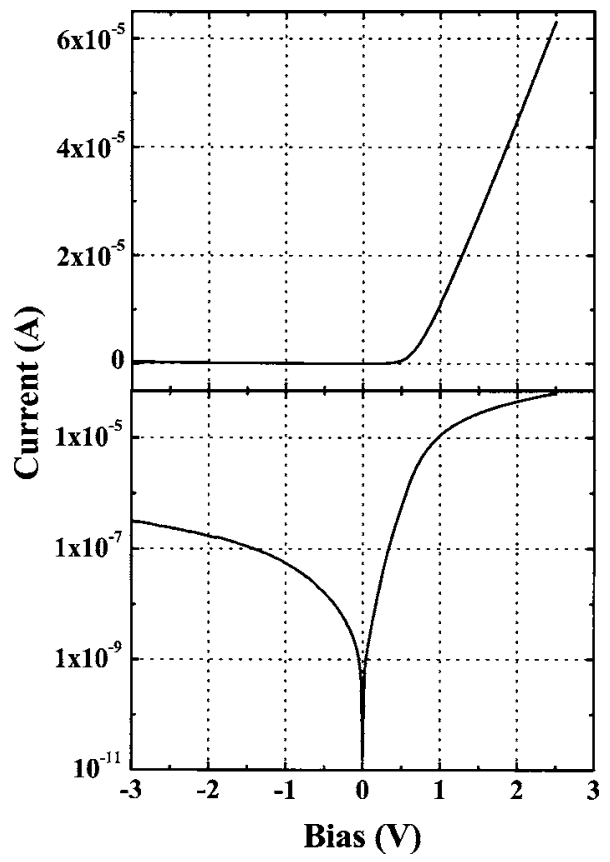

FIG. 1. Room-temperature $I-V$ characteristics of Au Schottky contact on nonpolar $a$ plane of $\mathrm{ZnO}$ in linear (top) and logarithmic (bottom) scale.

for every temperature. Note that each line scan was conducted over a previously unexposed area to avoid the influence of electron irradiation. ${ }^{6}$

In addition to diffusion length measurements, temperature-dependent changes in carrier lifetime were monitored by recording CL spectra in the near-band-edge (NBE) region. A Gatan MonoCL3 CL system is integrated with the SEM, which allows temperature- and wavelengthdependent experiments to be conducted in situ. The peak intensity of the NBE luminescence was monitored to assess the variation in carrier lifetime with increasing temperature. Both EBIC and CL measurements were performed over the range of temperatures from $25^{\circ} \mathrm{C}$ to $125^{\circ} \mathrm{C}$. An accelerating voltage of $20 \mathrm{kV}$ was used. As for EBIC experiments, several spectra were recorded at every temperature, each at a new location, after which the mean value of the peak intensity was determined, along with the standard deviation.

Recent technological improvements in $\mathrm{ZnO}$ technology prompted vigorous efforts to produce Schottky contacts to $\mathrm{ZnO}$ with good rectifying characteristics. ${ }^{11-14}$ Generally, the challenges in this area are related to low breakdown voltage and high leakage current of such contacts. Figure 1 shows the current-voltage $(I-V)$ characteristics of $\mathrm{Au}$ Schottky contacts deposited on the nonpolar $a$ plane of $\mathrm{ZnO}$. Roomtemperature measurements revealed good rectifying behavior with a low leakage current of $\sim 0.3 \mu \mathrm{A}$ at the reverse bias of $3 \mathrm{~V}$.

Table I summarizes the results of EBIC measurements and shows that the diffusion length of minority holes in $n-\mathrm{ZnO}$ increases with increasing temperature, $T$. The increase of $L$ with $T$ is not unique for this semiconductor. Similar trends were previously observed in GaAs (Ref. 15) and later in GaN epitaxial layers. ${ }^{7}$ In all cases, this increase was exponential with temperature and was modeled with the following expression: ${ }^{15}$
TABLE I. Temperature dependence of minority carrier diffusion length and CL intensity of the NBE peak in $n$ - ZnO.

\begin{tabular}{ccc}
\hline \hline $\begin{array}{c}\text { Temperature } \\
\left({ }^{\circ} \mathrm{C}\right)\end{array}$ & $\begin{array}{c}\text { Diffusion } \\
\text { length }(\mu \mathrm{m})\end{array}$ & $\begin{array}{c}\text { CL intensity } \\
\left(10^{3} \text { counts }\right)\end{array}$ \\
\hline 25 & $0.438 \pm 0.022$ & $72.1 \pm 3.7$ \\
50 & $0.472 \pm 0.060$ & $54.4 \pm 3.8$ \\
75 & $0.493 \pm 0.028$ & $49.2 \pm 2.4$ \\
100 & $0.520 \pm 0.074$ & $44.6 \pm 4.7$ \\
125 & $0.547 \pm 0.086$ & $38.5 \pm 6.8$ \\
$E_{A}(\mathrm{eV})$ & $0.045 \pm 0.002$ & $0.058 \pm 0.007$ \\
\hline \hline &
\end{tabular}

where $L_{0}$ is a scaling factor, $E_{A}$ is the activation energy, and $k$ is the Boltzmann constant. Figure 2 shows the experimental results for $n-\mathrm{ZnO}$ obtained in this work and the fit using Eq. (1), which yields an activation energy of $45 \pm 2 \mathrm{meV}$. The latter parameter represents carrier delocalization energy, since it determines the increase of the diffusion length due to reduction of recombination efficiency (see discussion below). ${ }^{15}$ The smaller the activation energy, the more efficient the thermally activated escape of captured carriers at any fixed temperature.

The temperature-induced increase of $L$ may be attributable to the growing lifetime of nonequilibrium minority holes in the valence band, as illustrated by the following expression:

$$
L=\sqrt{D \tau}
$$

where $\tau$ is carrier lifetime and $D$ is the diffusivity. Note that the temperature dependence of diffusivity and its effect on diffusion length are addressed later in the letter.

The role of increasing carrier lifetime is supported by the results of CL measurements, which are presented in Fig. 3. The inset of Fig. 3 shows a CL spectrum in the vicinity of the NBE transition at $385 \mathrm{~nm}(3.22 \mathrm{eV})$. It was observed that the peak intensity, $I$, of NBE luminescence decays systematically with increasing temperature, providing direct evidence that the number of recombination events decreases. The decay proceeds exponentially according to the equation below: ${ }^{16}$

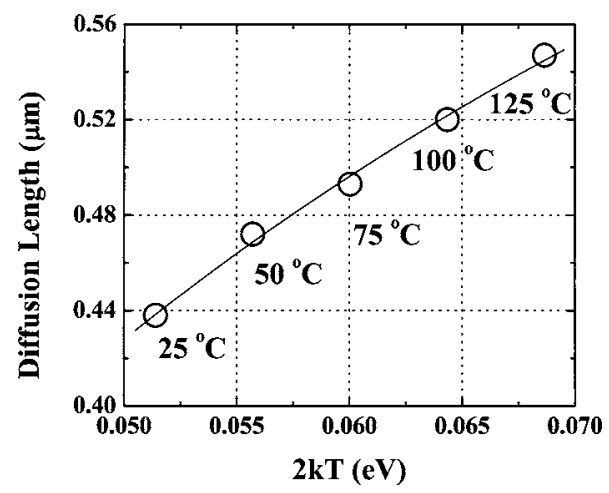

FIG. 2. Experimental dependence of minority carrier diffusion length on temperature (open circles). The line shows the fit [Eq. (1)] with activation energy of $45 \pm 2 \mathrm{meV}$. 


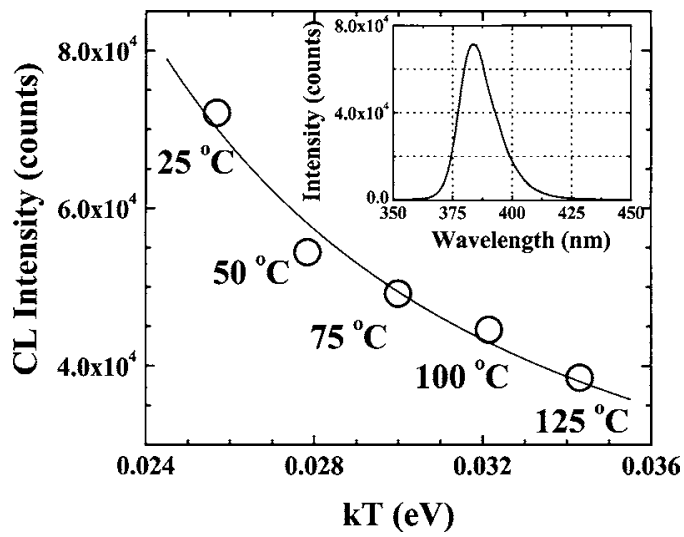

FIG. 3. Experimentally obtained values for the peak NBE CL intensity as a function of temperature (open circles) and the fit [solid line; Eq. (3)], yielding activation energy of $58 \pm 7 \mathrm{meV}$. Inset: CL spectrum on $n-\mathrm{ZnO}$ showing the NBE transition at $25^{\circ} \mathrm{C}$.

$$
I=\frac{A}{\left[1+B \exp \left(-\frac{E_{A}}{k T}\right)\right]},
$$

where $A$ and $B$ are scaling factors, and $E_{A}$ is the activation energy, similar in nature to that in Eq. (1). Based on the fit shown in Fig. 3, the activation energy was determined to be $58 \pm 7 \mathrm{meV}$. This energy is in reasonable agreement with that obtained by variable-temperature EBIC measurements, which suggests that the same underlying process is responsible for both the increase in the diffusion length and the CL intensity decay. This process is outlined below.

The increase in minority hole lifetime in the valence band is likely associated with a smaller recombination capture cross section for this carrier at elevated temperatures. In $\mathrm{GaAs}$, for example, a detailed analysis for the temperature dependence of the capture cross section indicates an order of magnitude decrease in recombination efficiency, measured in terms of an "effective capture radius", in the temperature range from 100 to $300 \mathrm{~K}^{15}$ Nonequilibrium electron-hole pairs are generated by the beam of the SEM, and subsequently annihilated by recombining with each other. Since the hole capture cross section is inversely proportional to temperature, ${ }^{15,17}$ the frequency of the recombination events (and, hence, the CL intensity) decreases as the temperature is raised. This means that nonequilibrium holes exist in the valence band for longer periods of time and, consequently, diffuse longer distances before undergoing recombination. Note that carrier diffusivity, $D$, is also a temperaturedependent quantity and, therefore, can affect the diffusion length. On the other hand, it has been demonstrated for $n$ - $\mathrm{ZnO}$ that the mobility, $\mu$, of the majority carriers decreases in the temperature range of our experiments by about a factor of $2 .^{18}$ Assuming that the mobility of the minority carriers exhibits the same behavior, ${ }^{7}$ and using the Einstein relation
( $D=\mu k T / q$, where $q$ is elementary charge), it is clear from Eq. (2) that the value of the diffusion length is dominated by the growing lifetime of minority holes. From the Einstein relation, the above-referenced difference in mobility translates to about a $30 \%$ decrease in diffusivity at $125^{\circ} \mathrm{C}$ as compared to $25^{\circ} \mathrm{C}$. Based on a $30 \%$ difference in diffusivity and using experimentally obtained values of diffusion length, we conclude that the lifetime of minority holes at $125^{\circ} \mathrm{C}$ is nearly 2.5 times greater than at room temperature.

In conclusion, temperature-dependent transport properties of minority carriers were studied in $n$ - $\mathrm{ZnO}$. The minority carrier diffusion length was observed to increase, while the CL intensity of the NBE transition decreased, indicating that the lifetime of nonequilibrium carriers in the band is greater at elevated temperatures. Activation energies obtained from both experimental techniques were in good agreement with each other, which suggests that both observations are manifestations of the same phenomenon.

This research was supported in part by the National Science Foundation (Grant No. ECS 0422604) and the American Chemical Society Petroleum Research Fund (Grant No. 40501-AC10).

${ }^{1}$ S. J. Pearton, D. P. Norton, K. Ip, Y. W. Heo, and T. Steiner, Prog. Mater. Sci. 50, 293 (2005).

${ }^{2}$ D. C. Look, Mater. Sci. Eng., B 80, 383 (2001).

${ }^{3}$ Y. I. Alivov, E. V. Kalinina, A. E. Cherenkov, D. C. Look, B. M. Ataev, A. K. Omaev, M. V. Chukichev, and D. M. Bagnall, Appl. Phys. Lett. 83, 4719 (2003)

${ }^{4}$ A. Osinsky, J. W. Dong, M. Z. Kauser, B. Hertog, A. M. Dabiran, P. P. Chow, S. J. Pearton, O. Lopatiuk, and L. Chernyak, Appl. Phys. Lett. 85, 4272 (2004).

${ }^{5}$ K. Ip, Y. W. Heo, D. P. Norton, S. J. Pearton, J. R. LaRoche, and F. Ren, Appl. Phys. Lett. 85, 1169 (2004);S. Y. Lee, E. S. Shim, H. S. Kang, S. S. Pang, J. S. Kang, Thin Solid Films 473, 31 (2005).

${ }^{6}$ O. Lopatiuk, W. Burdett, L. Chernyak, K. P. Ip, Y. W. Heo, D. P. Norton, S. J. Pearton, B. Hertog, P. P. Chow, and A. Osinsky, Appl. Phys. Lett. 86, 012105 (2005).

${ }^{7}$ L. Chernyak, A. Osinsky, H. Temkin, J. W. Yang, Q. Chen, and M. A. Khan, Appl. Phys. Lett. 69, 2531 (1996).

${ }^{8}$ H. J. Leamy, J. Appl. Phys. 53, R51 (1982).

${ }^{9}$ K. L. Luke, J. Appl. Phys. 80, 5775 (1996).

${ }^{10}$ H. K. Kuiken and C. Vanopdorp, J. Appl. Phys. 57, 2077 (1985).

${ }^{11}$ K. Ip, B. P. Gila, A. H. Onstine, E. S. Lambers, Y. W. Heo, K. H. Baik, D. P. Norton, S. J. Pearton, S. Kim, J. R. LaRoche, and F. Ren, Appl. Surf. Sci. 236, 387 (2004).

${ }^{12}$ R. Khanna, K. Ip, K. K. Allums, K. Baik, C. R. Abernathy, S. J. Pearton, Y. W. Heo, D. P. Norton, F. Ren, R. Dwivedi, T. N. Fogarty, and R. Wilkins, Phys. Status Solidi A 201, R79 (2004).

${ }^{13}$ S. H. Kim, H. K. Kim, and T. Y. Seong, Appl. Phys. Lett. 86, 112101 (2005).

${ }^{14}$ S. H. Kim, H. K. Kim, and T. Y. Seong, Appl. Phys. Lett. 86, 022101 (2005).

${ }^{15}$ M. Eckstein and H. U. Habermeier, J. Phys. IV 1, 23 (1991).

${ }^{16}$ D. S. Jiang, H. Jung, and K. Ploog, J. Appl. Phys. 64, 1371 (1988).

${ }^{17}$ J. I. Pankove, Optical Processes in Semiconductors (Prentice-Hall, Englewood Cliffs, New Jersey, 1971).

${ }^{18}$ D. C. Look, D. C. Reynolds, J. R. Sizelove, R. L. Jones, C. W. Litton, G. Cantwell, and W. C. Harsch, Solid State Commun. 105, 399 (1998). 